\title{
Enfermedades respiratorias por exposición a amianto, aspectos clínico-laborales y médico-legales
}

\author{
Respiratory disease occupational exposure to asbestos, medical clinical and \\ legal aspects
}

José Manuel Vicente Pardo

Unidad Médica Equipo Valoración Incapacidades Gipuzkoa. INSS. España.

Recibido: 26-08-14

Aceptado: 09-09-14

José Manuel Vicente Pardo

Pescadores Gran Sol s/n

20011 San Sebastián

Gipuzkoa. España.

Correo electrónico: jose-manuel.vicente@seg-social.es

Resumen

En este trabajo se pretende aclarar qué es y qué no es Enfermedad Profesional, en patologías relacionadas con la exposición laboral al amianto, en España, qué pruebas diagnósticas y de valoración funcional se precisan, qué comprobaciones de exposición han de realizarse y las consecuencias de su adscripción con carácter de enfermedad profesional.

Conclusiones: La enfermedad profesional está dotada de unas condiciones favorables de protección respecto de la enfermedad común, tanto económica de las prestaciones a que diera lugar como asistenciales o farmacéuticas para el trabajador que las padezca. Pero tiene una serie de consecuencias económicas que es preciso conocer como son el hecho de que la asistencia, y las prestaciones económicas de estos procesos corresponden y corren a cargo de la Mutua correspondiente y no al Servicio Público de Salud.

Puede implicar medidas sancionadoras a la empresa en el supuesto del incumplimiento por los empresarios de sus obligaciones en materia de prevención de riesgos laborales o cuando la enfermedad se produzca porque no se hayan observado las medidas generales o particulares de seguridad o higiene en el trabajo, o las de adecuación personal a cada trabajo.

La enfermedad profesional es atendida desde tres ámbitos normativos e institucionales diferentes el sistema sanitario, el preventivo y el de seguridad social que vertebra el concepto e implicaciones de las mismas. Las enfermedades profesionales tienen un especial tratamiento jurídico diferenciador RD 1299/2006, un especial tratamiento en la LGSS y la exposición laboral al amianto tiene además una norma específica de seguridad y salud en el trabajo. RD 396/2006. Debemos conocer la trascendencia de los informes médicos de valoración de la contingencia laboral de estos procesos y la cadena de sucesos jurídicos, concatenación de procedimientos y condenas judiciales, que en razón a la consideración de enfermedad profesional y la falta de medidas de seguridad puedan implicar.

Material y método: Se han revisado hasta febrero de 2014 las siguientes bases de datos bibliográficas: SciELO, y PUBMED. Así como la Bibliografía y documentos de consulta citados en este texto.

Med Segur Trab (Internet) 2014; 60 (236) 508-526

Palabras clave: Enfermedades respiratorias por exposición al amianto (asbesto), Enfermedad profesional. 


\section{Abstract}

This paper aims to clarify what is and is not Occupational Disease in pathologies related to occupational exposure to asbestos, in Spain, which diagnostic and functional assessment tests are required, what checks have been performed and show the consequences his assignment as an occupational disease.

Conclusions: Occupational disease is provided with favorable conditions for protection on the common disease, both economic benefits that would result as healthcare or pharmaceutical worker for the suffering. But it has a number of economic consequences that need to know such as the fact that assistance, and economic benefits of these processes are and are the responsibility of the relevant Mutual and not the Public Health Service. It may involve penalties to the company actions in the event of non-compliance by employers of their obligations regarding the prevention of occupational risks or when the disease occurs because they have not observed the general or specific measures of safety or occupational health or the personal suitability for the job. Occupational disease is serviced from three different policy and institutional areas the health system, preventive and social security structures the concept and implications thereof. Occupational diseases have a special legal treatment differentiator 1299/2006 RD, special treatment in LGSS and occupational exposure to asbestos also has a specific standard of safety and health at work. RD 396/2006. We know the importance of medical reports assessing occupational contingency of these processes and the chain of legal events, concatenating sentences and judicial procedures, which due to the consideration of occupational disease and the lack of safety measures might entail.

Med Segur Trab (Internet) 2014; 60 (236) 508-526

Keywords: Respiratory disease from exposure to asbestos, Occupational disease. 
El objetivo de este trabajo es aclarar qué es y qué no es Enfermedad Profesional de los procesos respiratorios por exposición al amianto, así como las pruebas precisas para su valoración funcional, en un intento de dar visibilidad a las Enfermedades Profesionales por exposición al amianto, sin perder el rigor en su acreditación.

\section{ENFERMEDADES RESPIRATORIAS POR EXPOSICIÓN A AMIANTO. CLASIFICACIONES}

A qué enfermedades nos referimos:

\subsection{Clasificación en dos grupos diferenciados entre cánceres y no cánceres.}

A) Enfermedades pleuropulmonares benignas por amianto

- Placas pleurales

- Fibrosis pleural difusa

- Derrame pleural benigno

- Atelectasia redonda o síndrome de Blesowski

- Asbestosis

B) Enfermedades pleuropulmonares malignas por amianto

- Mesotelioma

- Cáncer de pulmón

Pero el abanico de enfermedades respiratorias por exposición al amianto se estrecha en las que de estas son consideradas como enfermedad profesional

Clasificación en dos grupos diferenciados según grupo de enfermedad profesional en que se hallan encuadradas el de las enfermedades profesionales respiratorias y el grupo de los cánceres recogidos como enfermedad profesional.

\subsection{ERA Enfermedades Relacionadas con el Amianto}

El término ERA es el acrónimo de Enfermedades Relacionadas con el AMIANTO.

Como ERA se recogen enfermedades no solo respiratorias, sino todas en las que existe evidencia de vinculación en su origen con la exposición al amianto, afectando a diversos sistemas del organismo.

De ellas seis pueden considerarse benignas (las placas pleurales, el engrosamiento pleural, los derrames pleurales benignos, las atelectasias redondas, la asbestosis y la obstrucción crónica al flujo aéreo bronquiolar) y cuatro malignas (el mesotelioma pleural, el mesotelioma peritoneal, el carcinoma broncopulmonar y otras neoplasias relacionadas con el amianto).

Se consideran como "ERA» las siguientes alteraciones y enfermedades causadas por la inhalación de fibras de amianto:

- Sistema respiratorio

- Asbestosis

- Fibrosis de la pleura

- Engrosamiento pleural

- Atelectasias redondas o síndrome de Blesovsky

- Derrame pleural benigno

- Adenocarcinoma de pulmón

- Mesotelioma 
- Sistema gastrointestinal

- Mesotelioma peritoneal

- Otros tumores del tracto gastrointestinal Ca gástrico Ca Colón

- Fibrosis retroperitoneal

- Laringe Ca Laringe

- Sistema cardiovascular HTP

- Sistema Inmunológico Depresión Inmunológica

- Piel Cuernos cutáneos

- Más otros cánceres de causalidad no probada como el cáncer de riñón, mama u ovario

\subsection{Clasificación de Becklake 1998 enfermedades por amianto}

\begin{tabular}{lll}
\hline \multicolumn{1}{c}{ Tipo } & \multicolumn{1}{c}{ Órgano Afectado } & \multicolumn{1}{c}{ Enfermedad o Condición } \\
\hline No Maligna & Pulmón & Asbestosis \\
\cline { 2 - 3 } & & Enfermedad de la Pequeña Vía Aérea \\
\cline { 2 - 3 } & Pleura & Pronquitis Crónica \\
\cline { 2 - 3 } & & Placas pleurales \\
\hline Maligna & Pulmón & $\begin{array}{l}\text { Reacciones víscero-parietales que incluyen los derrames } \\
\text { atelectasias redondas }\end{array}$ \\
\cline { 2 - 3 } & Pleura & Cáncer pulmonar (todos los tipos) \\
\hline
\end{tabular}

\section{Controversia entre Criterios Médicos y Normativo-Jurídicos}

No existe unanimidad en cuanto a qué Pruebas Acreditan el Diagnóstico, de estos procesos. En lo que respecta a la Etiología C (concausa, multicausa, causa desconocida) la evidencia científica no mantiene los mismos niveles en las enfermedades relacionadas con la exposición al asbesto.

La controversia en materia de determinar la Etiología Laboral y No Laboral es importante, y no del todo común no tan solo en especialistas de medicina del trabajo, sino en las diversas normas que regulan esta adscripción en países del entorno.

Las Pruebas de Exposición del paciente al amianto y su nivel de garantía probatoria en cuanto a intensidad, tiempo de exposición, y constatación tienen como principal problema la falta de ellas en el momento en que se produjo la exposición años pasados.

La determinación del Nivel de Riesgo cuando hablamos de situación acaecida hace quince o veinte años es imposible de concretar, máxime si se trata de empresas desaparecidas, y lo mismo ocurre cuando intentamos establecer el Tiempo de exposición o las Tareas que desarrolló con exposición al amianto.

Así que navegamos en la incertidumbre en un contexto en que debiéramos establecer una Determinación Cierta y Objetiva.

\section{CLASIFICACIÓN Y CRITERIOS DE INCLUSIÓN COMO ENFERMEDAD PROFESIONAL. Enfermedades respiratorias por exposición al amianto en el Cuadro de Enfermedades Profesionales}

Como hemos referido las clasificaciones con inclusión de enfermedades en relación a la exposición al amianto son varias, pero el abanico se estrecha cuando de todas las anteriormente referidas nos ceñimos a aquellas que se encuentran en el Cuadro de 
Enfermedades profesionales actualmente en vigor recogido en el RD 1299/2006 de 10 de noviembre. La consideración de Enfermedad Profesional es un "constructo legal», una serie de normas que acotan su condición.

\subsection{Algoritmo de decisiones para la consideración de una enfermedad como enfermedad profesional}

El Art. 116 de Texto Refundido de la Ley General de Seguridad Social, RD 1/1994 de 20 junio, estable el Concepto de Enfermedad Profesional:

Se entenderá por enfermedad profesional «la contraída a consecuencia del trabajo ejecutado por cuenta ajena en las actividades que se especifiquen en el cuadro que se apruebe por las disposiciones de aplicación y desarrollo de esta Ley, y que esté provocada por la acción de los elementos o sustancias que en dicho cuadro se indiquen para cada enfermedad profesional".

Para la consideración de la Enfermedad Profesional desde esta consideración conceptual (art. 116 LGSS) de que debe ser enfermedad contraída consecuencia del trabajo, es decir una relación directa causal con el trabajo, en las actividades listadas en dicho cuadro y en relación con la presencia de un agente causal (el amianto) que es el que provoca la enfermedad, debe reunir estos condicionantes de forma simultánea:

1. Enfermedad referida en el Listado de Enfermedad Profesional. Anexo I. RD 1299/2006. Diagnóstico validado o cierto.

2. Contraída como resultado de la exposición a factores de riesgo inherentes a la actividad laboral. Datos que acrediten factores de riesgo inherentes a la actividad laboral.

3. Exposición al Amianto agente contaminante causal referido en el listado. Exposición y tiempo de exposición bien continuada o intensa al amianto. Debidamente constatada esta exposición tanto en concentración como en tiempo que se mantuvo la misma.

4. Actividad laboral listada con exposición a este agente (riesgo). Confirmación de actividad laboral desarrollada de las listadas.

Tratándose de darse los anteriores condicionantes de una Enfermedad Profesional a la que se le aplica el concepto jurídico de presunción «iuris et de iure» es decir no admite prueba en contrario. La presunción legal, característica del Sistema de Lista, libera al trabajador de "tener que probar» que su dolencia está causada por el trabajo.

Basta con probar por tanto, que es una enfermedad figura en la lista, que su actividad profesional lo pone en contacto con el agente nocivo generador de tal enfermedad, y que este agente está presente en su trabajo.

¿Qué es Enfermedad Profesional? (Art. 116 Ley General de la Seguridad Social) $\bigotimes$

- Enfermedad $\rightarrow$ contraída a consecuencia del trabajo

- En trabajos que se referencian en el listado, como de riesgo

- Provocada por la acción de elementos o sustancias que en dicho cuadro se indican para cada EP.

- Presente en el listado del RD 1299/2006 de 10 de noviembre, Anexo I.

\subsection{CUADRO DE ENFERMEDADES PROFESIONALES ANEXO 1:}

\section{Enfermedades profesionales relacionadas con amianto}

A) Grupo 4: "Enfermedades profesionales respiratorias causadas por inhalación de Polvo de Amianto".

- Enfermedades profesionales causadas por inhalación de Polvo de Amianto 
- Asbestosis

- Afecciones fibrosantes de la pleura y pericardio que cursan con restricción respiratoria o cardiaca provocadas por amianto

B) Grupo 6: “Enfermedades profesionales Cánceres por amianto»

- Enfermedades profesionales causadas por Amianto como agente carcinógeno

- Neoplasia maligna de bronquio y pulmón

- Mesotelioma

- Mesotelioma de pleura

- Mesotelioma de peritoneo

- Mesotelioma de otras localizaciones

\section{La lista de enfermedades del Anexo II Real Decreto 1299/2006}

Lista de Enfermedades Complementaria, cuyo origen profesional se sospecha pero no está científicamente probado ni constatado. (Podrían incluirse en el futuro en la lista de enfermedades profesionales del anexo I, si su origen profesional se prueba).

\section{Enfermedades provocadas por agentes carcinogénicos}

- Carcinoma de laringe producido por la inhalación de polvo de amianto

\section{CARACTERÍSTICAS DE LAS ENFERMEDADES PROFESIONALES POR EXPO- SICIÓN AL AMIANTO} tardío.

Tienen un Inicio lento, un desarrollo de la enfermedad lento y un debut clínico

Periodo de latencia largo, que pueden abarcar desde los 10-15 años para el cáncer de pulmón, los 15-20 para las placas pleurales o los 20-30 años para el mesotelioma.

En cuanto Intensidad de exposición, dosis y tiempo de exposición al amianto se conforman de forma variable; si parece tiene significación en la asbestosis pero no lo es tanto para el resto. En cuanto a los cánceres tienen una presentación estocástica o azarosa dependiendo menos de la intensidad, acúmulo de dosis o tiempo prolongado y continuado de exposición.

Son eso si enfermedades Previsibles. Se conoce lo que va a ocurrir si uno se expone al riesgo. Y cursaran de forma Progresiva si no se evitan los factores de riesgo desencadenantes o causales.

\subsection{Factores que determinan enfermedad profesional de las enfermedades respiratorias con origen en la exposición a amianto}

- Diagnóstico confirmado de Enfermedad dentro de las listadas Asbestosis, Fibrosis Pleural Ca. Bronco Pulmonar Mesotelioma

- Presencia de una Agente (Amianto) en las tareas que realiza

- Tiempo de exposición.

- Concentración del agente contaminante en el ambiente de trabajo.

- Tareas o actividad con demostrada exposición al Agente (Amianto)

- Características personales del trabajador

- Presencia de varios contaminantes o agentes causales al mismo tiempo. Multiplicidad de causas. Largo periodo de latencia

- Condiciones de seguridad. 


\subsection{Como el resto de las enfermedades profesionales tienen una serie de particularidades.}

3.2.1. Presunción "iuris et de iure" en la Enfermedad Profesional. Se presume siempre que se den requisitos legales. NO PERMITE PRUEBA EN CONTRARIO.

No es preciso, por tanto, la acreditación de la relación de causalidad entre el trabajo y la enfermedad.

3.2.2. Es de aplicación el periodo de observación de la incapacidad temporal, que se considera como el tiempo necesario para que, mediante el estudio médico se confirme o descarte un cuadro de EP. Si el trabajador está incapacitado para el trabajo cursará una IT ( 6 meses +6 meses de prórroga).

3.2.3. Puede acudirse a la figura de la sospecha de EP. Comunicación de enfermedades que podrían ser calificadas como Enfermedad Profesional. Art. 5 RD 1299/2006. "Los facultativos del SNS, con conocimiento de la existencia de una enfermedad cuyo origen profesional se sospecha, lo comunicarán a través del organismo competente de cada comunidad autónoma, a la Entidad Gestora, a los efectos de calificación y, en su caso, a la entidad colaboradora de la Seguridad Social que asuma la protección de las contingencias profesionales. Igual comunicación deberán realizar los facultativos del Servicio de Prevención, en su caso".

3.2.4. Podría considerarse el traslado de puesto. Si en un Reconocimiento Médico se descubre algún síntoma de enfermedad profesional que no constituye Incapacidad temporal, pero cuya progresión es posible evitar mediante el traslado del enfermo a otro puesto de trabajo exento de riesgo, se deberá llevar a cabo el traslado dentro de la misma empresa, sin que este cambio afecte a las retribuciones que percibe el trabajador (excepto complementos) art 133 LGSS.

\subsubsection{Otros factores en la EP con actuación preventiva.}

Incumplimiento de medidas de Seguridad e Higiene en el Trabajo puede dar lugar al Recargo de prestaciones.

\section{La Responsabilidad del empresario prevención de riesgos laborales.}

La Ley de Prevención de Riesgos Laborales establece en su artículo 14.1, «Derecho a la protección frente a los riesgos laborales". "Deber del empresario de protección de los trabajadores frente a los riesgos laborales«. «El incumplimiento por los empresarios de sus obligaciones en materia de prevención de riesgos laborales dará lugar a Responsabilidades Administrativas, así como, en su caso a responsabilidades Penales y a las Civiles por daños y perjuicios que puedan derivarse de dicho incumplimiento".

Recargo de prestaciones económicas del sistema de la Seguridad Social FMS Artículo 123 LGSS:

Cuando la enfermedad se produzca porque no se hayan observado las medidas generales o particulares de seguridad o higiene en el trabajo, o las de adecuación personal a cada trabajo, habida cuenta de las características del trabajador, las prestaciones económicas que tengan causa en la Enfermedad Profesional así contraída se aumentarán, según la gravedad de la falta, de un 30 a un 50 por cien.

Protocolo de Vigilancia Sanitaria Específica del Amianto y Protocolo de Vigilancia de la Salud de Trabajadores Expuestos a Amianto (CCAA)

Tienen como finalidad cumplir con el deber de vigilancia de la salud de los trabajadores que han estado expuestos al amianto, constituir la cohorte de trabajadores expuestos a amianto en cada Comunidad Autónoma y realizar estudios epidemiológicos con el fin de valorar el efecto del amianto en la salud de los trabajadores y su impacto en la comunidad. 


\title{
Vigilancia de la salud de trabajadores expuestos a Amianto
}

\author{
Separación del trabajo + IT por EP (período de observación y asimilados) si \\ presentara:
}

- Disnea de esfuerzo.

- Dolor torácico persistente no atribuible a otro tipo de patología.

- Crepitantes inspiratorios persistentes ("velcro"), basales o axilares.

- Alteraciones radiológicas pleurales no filiadas o de nueva aparición, o alteraciones radiológicas sospechosas de enfermedad pulmonar intersticial difusa.

- Alteraciones de la exploración de la función ventilatoria restrictiva compatibles con patología.

La Enfermedad Profesional es señal inequívoca de un fracaso de las medidas de prevención. Por lo que tiene de proceso "de conocida y directa causalidad". Pero la «norma» expresa para su regulación sucede con posterioridad a la constatación de causalidad médica probada, en el caso del amianto ya desde 1927 (Cooke) se conocía el perjudicial efecto del uso del amianto y la regulación más antigua al efecto en España data de 1940.

\section{ASPECTOS NORMATIVOS}

\subsection{Normas, instrucciones, Protocolos, Guías, Convenio}

Aunque la normativa e instrucciones son abundantes al respecto desde la Orden 31 de enero de 1940, que era de aplicación a la actividad industrial con exposición al amianto aunque regulada de forma genérica, como actividad peligrosa, no fue hasta que la Orden de 7 de diciembre de 2001 cuando se prohíbe la comercialización y uso del amianto.

Prohibición del amianto en España que fue progresivamente implantándose desde 1984 hasta 2001 a través de los siguientes textos legales:

Orden de 31 octubre de 1984 por la que se aprueba el reglamento sobre trabajos con riesgo de amianto.

Real Decreto 1406/1989 de 10 de noviembre de 1989, por el que se imponen limitaciones a la comercialización y al uso de ciertas sustancias y preparados peligrosos.

Orden 30 diciembre 1993: Productos químicos. Actualiza el anexo del Real Decreto 1406/1989, de 10 noviembre, que impone limitaciones a la comercialización y uso de ciertas sustancias y preparados peligrosos.

Orden Ministerial del 07/12/2001 por la que se modifica el anexo I del Real Decreto 1406/1989 del 10 de noviembre, por el que se imponen limitaciones a la comercialización $\mathrm{y}$ al uso de ciertas sustancias y preparados peligrosos.

Prohibición expresa de la comercialización de crocidolita, amosita, antofilita, actinolita y tremolita y de los productos que las contengan intencionadamente. Prohibición de la comercialización y utilización del crisotilo y de los productos que contengan esta fibra añadida intencionadamente. No obstante se podrá utilizar en los diafragmas destinados a instalaciones de electrólisis ya existentes, hasta que alcancen el fin de su vida útil o hasta que se disponga de sustitutivos adecuados sin amianto. El uso de productos que contengan las fibras de amianto que ya estaban instaladas o en servicio antes de la fecha de entrada en vigor de la presente Orden, seguirá estando permitido hasta su eliminación o el fin de su vida útil. Esta orden prohíbe definitivamente (a excepción de crisotilo en diafragmas destinados a instalaciones de electrólisis) la utilización, producción y comercialización de amianto, entrando en vigor dicha Orden el 
14/06/2002, con un periodo de permisividad de comercialización de los stocks de fabricación de 6 meses (hasta 14/12/2002).

Como colofón normativo en el RD 396/2006, de 31 de marzo, se establecen las disposiciones mínimas de seguridad y salud aplicables a los trabajos con riesgo de exposición al amianto.

En las diversas comunidades se fueron desarrollando diversas normativas, para facilitar la declaración de la enfermedad profesional.

Por otra parte los protocolos de vigilancia a nivel nacional se han ido actualizando como ocurre con El Protocolo de Vigilancia Sanitaria Específica 3. ${ }^{a}$ edición 2013.

Así mismo se han ido sucediendo Convenios de Amianto del INSS con las comunidades autónomas como el suscrito con el Gobierno Vasco en 2012 y el Convenio Amianto INSS Comunidad Autónoma de Madrid. Estableciendo Datos Mínimos para Valoración de las enfermedades por amianto y de los trabajadores afectos, Procesos que vigila, Población diana, y Pruebas para su determinación.

También ha contribuido al esclarecimiento de la enfermedad los Archivos de Registro tanto de EMPRESAS conocido como RERA es el registro de empresas con riesgo amianto, como el de TRABAJADORES conocido como RETEA registro de trabajadores expuestos al amianto

\subsection{Aspectos controvertidos de la legislación}

Señalábamos que tras muy diversas normativas la primera del año 1940, que regulan y restringen el uso del amianto será finalmente, en 2001 cuando se traspone en España la Directiva 1999/77/CE y se establece la probibición absoluta de la comercialización y utilización de fibras de cualquier variedad de amianto y de los productos que la contuvieran que se hace firme en 2002, y que la regulación del as enfermedades profesionales por exposición al amianto se efectúa en el RD 1299/2006, incluyendo normativa para la notificación y el registro de las mismas.

Pero esta larga carrera de normas que restringen y regulan el uso del amianto y diversas acciones preventivas nos ponen sobre aviso de que tanto las fecha restricción y prohibición son relativamente cercanas y marcan un tiempo en el que se estuvo expuesto al amianto, habida cuenta además de su uso extensivo en múltiples materiales y su riesgo en múltiples actividades, y por otra parte, advertir que las enfermedades consecuencia de estar expuesto a amianto tienen un largo periodo de latencia que inducen a pensar con alerta que en la aparición de enfermedades por amianto no hemos tocado techo y se prevé un pico entre los años 2015 y 2020.

Aún y con todo continúan existiendo deficiencias de su notificación, infradeclaración, pues se constata la existencia de más casos que los declarados oficialmente (incluidos en CEPROSS).

El procedimiento de archivo y control es poco eficiente, por vinculación insuficiente entre el profesional médico con competencia para calificar la contingencia, y el que asiste al paciente y debiera elevar la sospecha.

Entre otras razones por la patología específica que aflora clínicamente de forma tardía en pacientes que estuvieron expuestos muchos años antes y que afecta a edad avanzada en ocasiones con comorbilidad y como expresión propia del daño de salud propio de la edad.

A pesar de las medidas para un mejor control del amianto y las campañas divulgativas y normas expresas para paliar los efectos de las enfermedades por exposición al amianto, persiste la "contaminación" por escasa conciencia del peligro, en tareas de rehabilitación "doméstica" en actividades de construcción en viviendas particulares o reformas de naves industriales, y tal vez un cierto temor a declarar la enfermedad en estos tiempos de precariedad laboral. 
Por último en nada favorece el correcto abordaje de los trabajadores afectados cuando existen compartimentos estancos de la información sanitaria entre instituciones

\section{VALORACIÓN MÉDICO LABORAL DE LAS ENFERMEDADES RESPIRATORIAS PRODUCIDAS POR EXPOSICIÓN AL AMIANTO Y SU INCAPACIDAD RESULTANTE.}

De forma genérica valorar una situación de incapacidad laboral supone conocer las capacidades perdidas (qué capacidades mantiene) y las capacidades requeridas (qué capacidades precisa), procediendo para la valoración de la capacidad laboral de la siguiente manera:

a) Determinar la existencia de una lesión o proceso patológico. Las Deficiencias, son el cuadro diagnóstico incapacitante, desarrollado con el estadiaje o gradación que proceda.

b) Objetivar sus consecuencias las Limitaciones orgánicas y/o Funcionales que origina en el trabajador.

Con lo anterior estaremos conociendo las capacidades funcionales del trabajador

c) Conocer las Tareas realizadas por el trabajador. Conocer los Requerimientos Profesionales y circunstancias específicas del ambiente laboral.

Con ello estaremos conociendo las capacidades funcionales que se precisan para desarrollar un trabajo.

d) Finalmente establecer un Juicio Clínico Laboral cotejando las capacidades restantes del trabajador y los requerimientos del trabajo que desarrolla.

La valoración funcional de la incapacidad es esencialmente multiaxial, pues precisa de valoración:

I. DIAGNÓSTICA DE SECUELAS: Se deberán valorar los diagnósticos impeditivos laborales o incapacitantes que constituyen las Deficiencias, son Diagnósticos desarrollados, no solo el estricto diagnóstico codificado del parte de baja sino que describen la situación de enfermedad o lesión por eso amplían su nominación incluyendo etapificación, estadiaje o clasificación o gradación funcional del proceso, así mismo incorporarán la Intervención quirúrgica si esta ha supuesto una amputación o modificación funcional o anatómica de la arquitectura corporal o biológica del organismo.

II. TERAPÉUTICA: Tratamientos recibidos, en curso o por recibir, y su servidumbre terapéutica* (tratamiento crónico, efectos secundarios, tratamiento prolongado durante determinado tiempo, cruento o no cruento, posibles indicaciones terapéuticas en un futuro, etc.).

III. EVOLUTIVA: En el curso del proceso, pronóstico (favorable, incierto, reversible, desfavorable, irreversible, pronóstico vital). Respuesta a los tratamientos y la propia deriva evolutiva del proceso en el tiempo.

IV. FUNCIONAL LIMITACIONES ORGÁNICAS, O FUNCIONALES: Obliga a jerarquizar las limitaciones funcionales, a graduar las limitaciones, a definir su intensidad y a referenciarlas respecto de las diversas funciones físicas o psíquicas de un individuo. Así como establecer aquellas funciones o funcionalidad que mantiene para poder describir y valorar la capacidad funcional restante.

V. DE REVISIÓN: no previsible mejoría, previsible mejoría de pendiendo de, previsión de mejoría notable, proceso de evolución incierta.

VI. PREVENTIVA: Tanto por el carácter del trabajo con tareas que estén contraindicadas con su enfermedad, pues pudieran ocasionar una agravación de la misma o aminorar o perjudicar la respuesta evolutiva favorable al tratamiento, como por el riesgo que el desarrollo del trabajo con sus limitaciones funcionales pudiera entrañar para sí o para otros. El carácter preventivo incapacitante domina 
en la contingencia de enfermedad profesional, donde caso de no poder aislar o apartar al trabajador del riesgo derivado de la exposición al agente causal de la enfermedad condicionaría la declaración de la incapacidad para ese trabajo.

* Servidumbre Terapéutica* es la pérdida de la autonomía funcional que ocasiona a un paciente el tratamiento. Derivada de los efectos secundarios de los tratamientos o la sujeción a tener que seguir un tratamiento determinado como sucede por ejemplo con la obligación en diálisis de estar indispuesto funcionalmente durante unas horas, para poder seguir el tratamiento adecuadamente y la necesidad de acudir a un centro hospitalario o estar sujeto a la terapia en su domicilio causando una indisposición de su autonomía funcional durante un tiempo y otros condicionantes añadidos como necesidad de desplazamiento o de ayuda sanitaria, etc.

\section{Peculiaridades Incapacidad Permanente en la Enfermedad Profesional}

Es en el único supuesto en que se determina la Incapacidad en base al carácter preventivo benéfico del alejamiento del trabajo, y no se precisa como en el resto de los supuestos (resto de contingencias) la presencia de lesiones y limitaciones funcionales.

Es decir incapacita la constatación diagnóstica ocupacional, caso de que la incapacidad y el alejamiento de la profesión sean la única posibilidad de evitar la reaparición de los síntomas o la cronificación del proceso o su agudización.

\section{Criterios de valoración de la incapacidad en el INSS}

Valorar la situación funcional en relación con respecto a la clínica que presenta, los hallazgos en Pruebas Funcionales, el tratamiento seguido, tipo, evolución, ingresos, y su servidumbre Terapéutica. Valorar la comorbilidad con otros procesos incapacitantes, el Índice pronóstico o supervivencia en caso de procesos graves.

Proceder a la declaración de la Incapacidad Permanente en el grado que proceda cuando no es posible el cambio de puesto de trabajo y se mantiene el trabajo con riesgo.

Valoración genérica en el caso especial de valorar trabajadores mayores de 65 años jubilados, lo que es posible en caso de la enfermedad profesional como excepción.

La calificación de las enfermedades como profesionales, a efectos de prestaciones de la Seguridad Social, corresponderá al INSS.

\section{Procesos específicos}

\subsection{Placas pleurales (CIE-9: 511.0)}

No es enfermedad incluida en listado de Enfermedades Profesionales:

- NO CALIFICABLE por tanto COMO Enfermedad Profesional.

- No conlleva disfunción, acostumbra a ser un hallazgo casual.

- Precisa de una Acción de Vigilancia para detectar otras patologías causadas por exposición amianto.

Indica exposición al amianto, es un testigo y marcador de exposición:

- Si se asocia placa pleural (CIE-9: 511.0) con trabajos que incluyan amianto y se excluye otra etiología, sería calificable como lesión asimilada a ENFERMEDAD DEL TRABAJO calificable como AT (art. 115.2.e).

- Declarable en PANOTRATSS (Archivo de patologías no traumáticas causadas o agravadas por el trabajo. En apartado 10 e, de Enfermedades del sistema respiratorio: Otras enfermedades de la pleura.

\subsection{Asbestosis. Sí es Enfermedad Profesional}

Fibrosis Pulmonar Intersticial Difusa secundaria a la inhalación de fibras de Asbesto. Los elementos básicos para el diagnóstico son los antecedentes de exposición al 
amianto, los hallazgos clínicos y radiológicos. Es una neumoconiosis no distinguible por la clínica de otras.

Diagnóstico según:

- Antecedente de exposición a asbesto en historia laboral. Tiempo de exposición $y$ actividad

- Hallazgos clínicos

- Hallazgos radiológicos sugestivos de fibrosis pulmonar difusa

- Hallazgos funcionales sugestivos de enfermedad Intersticial Difusa

- Tiempo de latencia adecuado > 15 años

Pruebas Diagnósticas

- La RX de tórax instrumento básico en la identificación de la enfermedad, no obstante el TAC de alta resolución ha añadido una mayor sensibilidad. (TACAR)

Pruebas de Valoración Funcional

- Las pruebas de función respiratoria muestran alteraciones ventilatorias restrictivas con disminución de la difusión pulmonar, con ellas determinamos el Grado Funcional de limitación y sus consecuencias incapacitantes

- Espirometría forzada tiene un valor diagnóstico y funcional relevante

- $\mathbf{D L C O}=$ Test de Capacidad de difusión de Monóxido de Carbono CO

- Pletismografía Disminución volúmenes pulmonares.

Pruebas de exposición

- La determinación de cuerpos de asbesto en BAL (lavado broncoalveolar) es un indicador de exposición aunque su ausencia no descarta enfermedad

ASBESTOSIS y la consideración de Incapacidad

- Valorar disfunción pulmonar y la disnea

- Puede ser causa de IPT x EP para actividades de contenido físico marcado

- Pero puede ser no causa de IPT y si condicionar acciones de seguimiento y vigilancia tanto ocupacional como postocupacional

5.3. Afecciones fibrosantes de la pleura o pericardio que cursan con restricción respiratoria o cardiaca. Sí son Enfermedad Profesional

- Pruebas diagnósticas y de valoración similares a la asbestosis para la afección pleural.

- Pruebas cardiacas ECG ECO para las afecciones pericárdicas

- Normalmente No son causa de IPT por EP, pero pueden objetivarse limitaciones funcionales que si condicionen IPT, e incluso dar lugar a IPA.

\subsection{Cáncer de pulmón sí puede ser Enfermedad Profesional}

Clínicamente el cáncer de pulmón debido a la exposición al amianto no se diferencia del de otro origen, por lo que será necesario probar la exposición al amianto en el desarrollo de su trabajo. Puede ir unido a asbestosis previa aunque no siempre.

La crocidolita es el más cancerígeno de los amiantos.

La incidencia aumenta con altos niveles de exposición aún en cortos periodos, aunque muestra un marcado incremento según aumenta la duración de exposición.

El riesgo de contraer un Ca de pulmón es mayor en trabajadores expuestos si además fuman. La exposición al amianto incrementa 5 veces el riesgo de cáncer de pulmón en los no fumadores y 55- 60 veces en los fumadores. 
La Asociación del Amianto y el Cáncer de Pulmón es diversa.

El efecto carcinogénico depende de la fibrosis pulmonar. La presencia de placas pleurales o de obstrucción al flujo aéreo también se asocia a un aumento del riesgo de cáncer de pulmón. La asbestosis no es un requisito indispensable para el efecto carcinogénico del Amianto en el Pulmón.

Las mutaciones para el protooncogén K-ras en tejido pulmonar de pacientes con adenocarcinoma pulmonar, ha demostrado una asociación entre la exposición al amianto. Se ha detectado que la exposición al amianto aumenta el riesgo de cáncer de pulmón. Aumento incidencia de cáncer de pulmón en individuos expuestos de forma ambiental a tremolita y crisotilo.

Aceptando relación directa entre exposición al Amianto y Ca Pulmón, las relaciones entre la dosis y la respuesta varían sustancialmente, el riesgo de desarrollar cáncer por exposición al amianto está relacionado con la exposición acumulada, la industria y el tipo de fibra.

En varios estudios se ha demostrado un riesgo más elevado en personas con una alta exposición y una sinergia sustancial con el tabaco

\section{Causalidad del AMIANTO del CÁNCER de PULMón}

Se debe aumentar la sospecha clínica del amianto como un factor potencialmente causante del cáncer de pulmón. Para probar la causalidad del amianto, resulta esencial la utilización de cuestionarios sistematizados y, siempre que sea posible, la determinación del amianto en pulmón.

\subsection{Cáncer de bronquio y pulmón y la valoración de la incapacidad}

Tumores T1a T1b T2a T2b NO o N1 dependiendo la funcionalidad restante pulmonar pueden ser Incapacidad Permanente Total por Enfermedad Profesional, habitualmente son Incapacidad Permanente Absoluta.

\subsection{Cáncer de la pleura si puede considerarse enfermedad profesional (mesotelioma pleural maligno):}

Es un tumor poco frecuente, tiene un período de latencia de más de 20 años.

La crocidolita es la variedad de amianto que más mesotelioma produce.

Su incidencia probablemente se incrementará en los próximos años debido al uso del amianto desde 1945 a 1970, el pico de consumo entorno a 1980 y el prolongado período de latencia, de más de 30 años.

Sospecha diagnóstica de mesotelioma

- Dolor torácico

- Derrame pleural

- Crecimiento en el grosor o aspecto festoneado de las placas pleurales

- Dolor en pacientes con placas asintomáticas previas

- Historia laboral de riesgo y período de latencia

- Estudio radiológico de imagen TACA

Diagnóstico confirmado:

- El diagnóstico definitivo se consigue a través de la biopsia pulmonar abierta o por toracoscopia.

- La determinación de cuerpos de amianto es útil para valorar los antecedentes de exposición. Estos cuerpos se pueden determinar en el esputo pero solo tienen una sensibilidad del 36\%, mientras que en el lavado bronco-alveolar la sensibilidad es del $85 \%$. 
- Una historia laboral bien dirigida, que apoye el diagnóstico.

Pronóstico: Es malo, con una mortalidad del 100\% a los 24 meses

\subsubsection{Mesotelioma y la valoración de la incapacidad}

Condiciona siempre una incapacidad permanente absoluta dado el pronóstico y la no posibilidad de curación, además de valorar la contingencia como enfermedad profesional cuando queda probada la exposición al amianto en el desarrollo de su trabajo. La población afectada, por lo general, tiene edades superiores a los 60 años lo que a menudo es la razón para que no se le asigne la causalidad laboral, quedando lejano el origen de la exposición, y la dificultad para relacionar la enfermedad con el trabajo, cuando la historia de exposición es muy lejana y desconocida o no concluyente. Mayor dificultad cuando se trata de pacientes de más de 75 años con comorbilidad severa con otras patologías.

Por todo ello, existe una gran dificultad para señalar los antecedentes probados de exposición en las enfermedades por amianto. Frente a los problemas de reconocer el antecedente de exposición al amianto mediante una entrevista clínica abierta o el interrogatorio clínico no sistematizado con una sensibilidad escasa es de aplicación recomendad la práctica de un cuestionario estructurado.

Entre las causas de esta baja sensibilidad se pueden apuntar diversos factores. El extenso tiempo de latencia entre exposición y enfermedad es uno de ellos, ya que dificulta el recuerdo preciso de los antecedentes por parte del paciente en lo que afecta a los productos y actividades desarrolladas.

\subsection{Actividades especificadas en el cuadro enfermedades profesionales por exposición al amianto. RD 1299/2006. Anexo I}

Trabajos de extracción, manipulación y tratamiento de minerales o rocas amiantíferas, minerales o rocas amiantíferas.

Fabricación de tejidos, cartones y papeles de amianto.

Tratamiento preparatorio de fibras de amianto de fibras de amianto (cardado, hilado, tramado, etc.).

Aplicación de amianto a pistola a pistola (chimeneas, fondos de automóviles y vagones).

Trabajos de aislamiento térmico en construcción naval y de edificios y su destrucción.

Desmontaje y demolición de instalaciones que contengan amianto.

Fabricación de guarniciones para frenos y embragues, de productos de fibrocemento, de equipos contra incendios, de filtros y cartón de amianto, de juntas de amianto y caucho.

Carga, descarga o transporte de mercancías que pudieran contener fibras de amianto.

\subsection{Sectores de actividad en los que de forma más directa los trabajadores están expuestos a fibras de amianto}

Construcción.

Industria del automóvil.

Industria de fabricación y mecanizado de productos de fibrocemento.

Industria de fabricación y utilización de juntas.

Industria naval. Industria ferroviaria.

Industria textil. 
Transporte, tratamiento y gestión de residuos.

Los dos tipos de operaciones en los que los trabajadores corren un riesgo más elevado son:

a) Operaciones de desamiantado (retirada de amianto).

b) Operaciones de reparación y mantenimiento de edificios, instalaciones industriales o maquinaria o barcos, trenes, etc. que contienen amianto.

\section{PARA LA DETERMINACIÓN DE UNA ENFERMEDAD POR LA EXPOSICIÓN LABORAL AL AMIANTO CANCEROSA O NO COMO ENFERMEDAD PROFESIONAL DEBEMOS CONSTATAR}

- Intensidad mínima de exposición:

- Exposición profesional confirmada por la anamnesis y evaluada por el examen de las condiciones laborales constatando la misma, que pongan en evidencia una exposición prolongada o suficiente y a ser posible objetivada por medidas ambientales y biológicas.

- Confirmación diagnóstica de la enfermedad:

- No vale la mera sospecha por una exposición a un agente cancerígeno presente en el trabajo.

- Realizar pruebas específicas para cada proceso.

- Criterios científicos de causalidad y asociación Enfermedad - Amianto.

- Requisitos para dar por suficientemente probada la exposición.

- Confirmación se ha estado expuesto:

- Pruebas de exposición en el tejido pulmonar.

- Pruebas de exposición en el medio laboral.

- Dosis, tiempo de exposición, tipo de actividad, tipo de amianto, nivel de riesgo

\section{SITUACIONES ESPECIALES EN ESTE TIPO DE PROCESOS}

\section{La Sospecha de Enfermedad Profesional sin situación de baja}

Desde el Médico de Atención Primaria del Servicio Público de Salud cuando se sospecha en el seguimiento de un paciente este presenta una posible Enfermedad Profesional.

Desde Servicio de Prevención cuando se sospecha en el seguimiento de un paciente este presenta una posible Enfermedad Profesional.

SI NO SE ACTÚA estamos propiciando el que un proceso no obligue a establecer protocolos de vigilancia que si se deben hacer cuando una enfermedad tiene la consideración de Enfermedad Profesional.

Además estamos facilitando el que la enfermedad pueda proseguir hasta que al final si sea incapacitante, es decir, termine en Incapacidad Terminal.

\section{Cambio de puesto de trabajo EP}

Reconocimientos médicos, cambio de puesto de trabajo y obligaciones normativas.

Todas las empresas que deban cubrir puestos de trabajo con riesgo de enfermedad profesional, están obligadas a practicar, a su cargo, reconocimientos médicos previos a la admisión de quienes vayan a ocuparlos, así como a realizar los reconocimientos periódicos 
que para cada tipo de enfermedad se establezcan en las normas. Estos reconocimientos son obligatorios y gratuitos para los trabajadores. Las empresas no podrán contratar trabajadores que no hubieran sido declarados aptos en el reconocimiento o permitir la continuación en su puesto de trabajo en el caso de que esta inaptitud reflejada fuera descubierta en los reconocimientos periódicos. El incumplimiento de estas obligaciones por parte de la empresa, la convierte en responsable directa de todas las prestaciones económicas que pueden derivarse en tales casos de la enfermedad profesional desarrollada y determinada su contingencia. Las Mutuas de Accidentes de Trabajo y Enfermedades Profesionales de la Seguridad Social están también obligadas a llevar un libro de reconocimientos médicos donde se inscriben los resultados de los efectuados a los trabajadores con riesgo de enfermedad profesional.

El traslado de puesto de trabajo es una medida protectora especial para la EP art. 45 OM de 9 mayo 1962. En caso de que no sea posible el cambio de puesto de trabajo los artículos 48 y 49 establecen una serie de medidas.

En los casos en que como consecuencia de un reconocimiento médico se descubra algún síntoma de EP que no constituya IT pero cuya progresión sea posible evitar mediante el traslado del enfermo a otro puesto de trabajo exento de riesgo, se deberá llevara a cabo el cambio de puesto de trabajo dentro de la misma empresa, sin que este cambio, afecte a las retribuciones que viene percibiendo el trabajador (excepto complementos de calidad y cantidad) art 133.2 del TRLGSS.

Los médicos que en el ejercicio de sus funciones, descubran dicha situación informarán a la empresa de sí el cambio tiene carácter provisional o definitivo, debiéndose hacer también a la Autoridad Laboral y a la Entidad con al que esté concertado el seguro de AT y EP.

\begin{tabular}{lll}
\hline \multicolumn{1}{c}{ CANTIDAD } & \multicolumn{1}{c}{ TIEMPO DE SUBSIDIO } & \multicolumn{1}{c}{ RESPONSABLE } \\
\hline $\begin{array}{l}\text { Subsidio equivalente al salario } \\
\text { integro }\end{array}$ & 12 meses & A cargo de la empresa \\
\cline { 2 - 3 } & 6 meses & A cargo del ente asegurador \\
\cline { 2 - 3 } & 6 meses prorrogables por otros 6 & A cargo del régimen de desempleo \\
\hline & $\begin{array}{l}\text { El tiempo máximo de percepción } \\
\text { es de 30 meses }\end{array}$ & \\
\hline
\end{tabular}

En el supuesto de que el agente nocivo que genera la EP esté extendido en todo el ámbito de trabajo deben iniciarse los trámites necesarios para la declaración de la IP.

La Ley 51/ 2007 de 26 de diciembre, de Presupuestos Generales del Estado, para el año 2008 establece en su disposición adicional quinta una reducción en la cotización a la Seguridad Social, en los términos y condiciones que reglamentariamente se determinen en aquellos casos en que, por razón de EP, se produzca un cabio de puesto de trabajo en la misma empresa o el desempeño, en otra distinta, de un puesto de trabajo compatible con el estado del trabajador.

La valoración en materia de seguridad social de una incapacidad laboral determina una referencia obligada al profesión o trabajo, dejando de lado otros aspectos ajenos a la laboralidad del proceso.

Por otra parte el carácter de enfermedad profesional una vez acreditada que la enfermedad esta incluida en el cuadro de enfermedades profesionales, que se demuestre que se estuvo expuesto al amianto, y que se realizaba una actividad de las que aparecen en el listado se constituye como tal no solo con un carácter prestacional, dando origen a la prestación que sea temporal o permanente, sino que además se le dota de un carácter preventivo con aspectos obligados de prevención tanto ocupacional como postocupacional.

\section{Valoración civil o penal}

- Si ante las Salas de lo Social las demandas han ido increscendo en los últimos años, con una presión social importante que las impulsa. Reflejo de un problema 
real, opaco en muchas ocasiones, como es "el enfermar por el amianto». Las Audiencias Provinciales y Tribunales Superiores han ido dictando numerosas resoluciones por responsabilidad civil o responsabilidad patrimonial, ante demandas cada vez también crecientes.

- Desde el punto de vista médico pericial el daño debe ser valorado en toda su extensión, es decir tanto el diagnóstico, como los tratamientos seguidos, como la necesidad o no de hospitalización, como las pérdidas en la autonomía personal en todos los ámbitos, personal, familiar, social y laboral.

- Por lo tanto en estos supuestos y debido a que se juzgará el daño y sus consecuencias, bien para su responsabilidad económica al declarado culpable o responsable, bien para su pena impuesta al culpable o responsable, es preciso exponer todas las circunstancias, diagnóstico, ingresos, intervenciones quirúrgicas tratamientos, evolución pronóstico, así como finalmente se valorarán no solo las repercusiones laborales sino las de otras áreas de la persona y su entorno, incluyendo la repercusión de la enfermedad en su vida de ocio o familiar.

- Se debe proceder a realizar aquellas actuaciones médicas encaminadas a la evaluación de todos aquellos daños sufridos por la persona y producidos por un determinado hecho lesivo, que permitan al juzgador conocer las consecuencias exactas de la enfermedad.

- Recordar que se valoran tanto el Daño Personal Patrimonial o económico que engloba el Daño Emergente es decir los gastos médicos y paramédicos, como el Lucro Cesante, es decir la reducción de la capacidad productiva y social, como se valorará el Daño Personal Extrapatrimonial, constituido por el Dolor, el Quantum Doloris, los Daños Morales, el Perjuicio Estético, la Pérdida de Bienestar, las Actividades Recreativas o de Ocio, así como Otros Daños que se demuestren han existido y la repercusión a terceras personas.

- No olvidar aquellos aspectos preventivos en torno a la aparición de la enfermedad que también deben ser valorados, y en la causalidad expuesta en los informes, acreditar lo que afirmemos en este aspecto, tanto en el reflejo concluyente de la causas de aparición de la enfermedad como con la suficiencia de soporte técnico al respecto, de la contaminación con el amianto, en forma, en tiempo de exposición y en intensidad si se conociera.

\section{CONCLUSIONES}

- La enfermedad profesional está dotada de unas condiciones favorables de protección respecto de la enfermedad común, tanto económica de las prestaciones a que diera lugar como asistenciales o farmacéuticas para el trabajador que las padezca. Pero tiene una serie de consecuencias económicas que es preciso conocer como son el hecho de que la asistencia, y las prestaciones económicas de estos procesos corresponden y corren a cargo de la Mutua correspondiente y no al Servicio Público de Salud.

- Puede implicar medidas sancionadoras a la empresa en el supuesto del incumplimiento por los empresarios de sus obligaciones en materia de prevención de riesgos laborales o cuando la enfermedad se produzca porque no se hayan observado las medidas generales o particulares de seguridad o higiene en el trabajo, o las de adecuación personal a cada trabajo.

- La enfermedad profesional es atendida desde tres ámbitos normativos e institucionales diferentes el sistema sanitario, el preventivo y el de seguridad social que vertebra el concepto e implicaciones de las mismas. Las enfermedades profesionales tienen un especial tratamiento jurídico diferenciador RD 1299/2006, un especial tratamiento en la LGSS y la exposición laboral al amianto tiene además una norma específica de seguridad y salud en el trabajo. RD 396/2006. Debemos conocer la trascendencia de los informes médicos de valoración de la 
contingencia laboral de estos procesos y la cadena de sucesos jurídicos, concatenación de procedimientos y condenas judiciales, que en razón a la consideración de enfermedad profesional y la falta de medidas de seguridad puedan implicar.

- Recordar que en cuanto a la causa o causalidad el amianto es un contaminante

- LABORAL (presente en el trabajo y/o por el trabajo)

- NO LABORAL (doméstico, de ocio, ambiental).

Para finalizar este decálogo de cuestiones a plantearse frente al AMIANTO Y EL TRABAJO:

1. Establecer la constatación del riesgo y la exposición al amianto, (VLA determinaciones de control, etc).

2. Vigilancia de salud ocupacional de las enfermedades por amianto.

3. Comprobación en el registro de trabajadores expuestos a amianto. RETEA.

4. Comprobación en el registro de empresas con riesgo de amianto RERA.

5. La necesidad de control a trabajadores sensibles, el concepto de trabajador sensible LPRL 95.

6. Alerta frente al riesgo actual en la contaminación por amianto, sectores más peligrosos (desamiantado, actividades de mantenimiento y obras de reforma de edificios (tanto industriales, públicos como privados, incluyendo las obras de reforma en las casa o comunidades de vecinos), actividades de desguace de barcos, trenes y otros ingenios industriales).

7. La utilidad de las entrevistas laborales a los pacientes asistidos por los SPS y su utilidad demostrada en la detección de las enfermedades por amianto.

8. El amianto como contaminante laboral, formas de amianto y tipo, la friabilidad y su presencia ambiental o en otros medios no laborales.

9. Para el afrontamiento de la presunción de culpabilidad, debe plantearse con rigor la constatación de la exposición, tiempo de exposición, realización de tareas el rigor en la determinación del factor de riesgo por amianto.

10. Análisis de evidencia de exposición y las diversas enfermedades por amianto, relación o no causal dosis respuesta, factores ajenos, factores genéticos y el nuevo concepto de exposoma.

\section{REFERENCIAS BIBLIOGRÁFICAS}

1. Gilson JC. Asbestos. En: Parmeggiani L (ed.). Enciclopedia de Salud y Seguridad en el Trabajo. Madrid: Ministerio de Trabajo y Seguridad Social, 1989: 279-82.

2. Boletín Oficial del Estado Real Decreto 1299/2006 por el que se aprueba el cuadro de enfermedades profesionales en el sistema de la Seguridad Social y se establecen criterios para su notificación y registro. BOE núm 302 de 19/12/2006.

3. Boletín Oficial del Estado. Orden de 7 de diciembre de 2001 por la que se modifica el anexo I del Real Decreto 1406/1989, de 10 de noviembre, por el que se imponen limitaciones a la comercialización y al uso de ciertas sustancias y preparados peligrosos. BOE núm 299 de 14/12/2001.

4. Boletín Oficial del Estado. Orden de 26/7/93 del Ministerio de Trabajo y Seguridad Social, por la que modifica el Reglamento sobre Trabajos con riesgo de amianto y las normas complementarias. BOE núm. $188,5 / 8 / 93$

5. Boletín Oficial del Estado. Orden de 31/10/84 del Ministerio de Trabajo y Seguridad Social, Reglamento sobre Trabajos con riesgo de amianto. BOE núm. 267, 7/11/84 (corrección de errores BOE núm. 280, 22/11/84).

6. Boletín Oficial del Estado. Orden de $7 / 1 / 87$ del Ministerio de Trabajo y Seguridad Social, normas complementarias del Reglamento sobre Trabajos con riesgo de amianto. BOE núm. 13, 15/1/87. 
7. Boletín Oficial del Estado. Decreto $1995 / 78$ por el que se aprueba el Cuadro de Enfermedades Profesionales. BOE núm 203 de 25/8/1978.

8. Organización Mundial de la Salud. Detección precoz de enfermedades profesionales. Ginebra: La Organización, 1987.

9. Segarra F. Asbestosis, talcosis y otras silicatosis. En: Segarra F (dir.). Enfermedades broncopulmonares de origen ocupacional. Barcelona: Editorial Labor, 1985: 293-350.

10. Griffith DE, Miller EJ, Gray LD, et al. Interleukin-1-mediated release of interleukin-8 by asbestosstimulated human pleural mesothelial cells. Am J Respir Cell Mol Biol 1994; 10: 245-52.

11. Parmeggiani L. Neumoconiosis, clasificación internacional. En: Parmeggiani L (ed.). Enciclopedia de Salud y Seguridad en el Trabajo. Madrid: Ministerio de Trabajo y Seguridad Social, 1989: 323-29.

12. García AM, Gadea R, López V. Estimación de la mortalidad atribuible a enfermedades laborales en España, 2004. Rev Esp Salud Pública 2007;81:261-270.

13. Montserrat García Gómez, Alfredo Menéndez-Navarro, y Rosario Castañeda López. incidencia en españa de la asbestosis y otras enfermedades pulmonares benignas debidas al amianto durante el período 19622010 Revista Española Salud Pública 2012; 86: 613-625.

14. Guía técnica para la evaluación y prevención de los riesgos relacionados con la exposición al amianto. Real Decreto 396/2006, de 31 de marzo. BOE n. ${ }^{\circ}$ 86, d 11 de abril.

15. Guía de buenas prácticas para prevenir o minimizar los riesgos del amianto en los trabajos en que esté presente (o pueda estarlo), destinada a empresarios, trabajadores e inspectores de trabajo, publicada por el comité de altos responsables de la inspección de trabajo (SLIC) de la Comisión Europea.

16. Programa integral de vigilancia de la salud de los trabajadores que han estado expuestos al amianto y protocolo de vigilancia sanitaria especifica del Ministerio de Sanidad y Consumo (versión 2003).

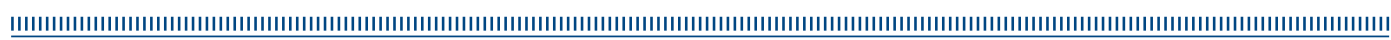

